vaccination of high-risk patients reduced winter hospitalizations, emergency room visits, and tests for respiratory ailments by $10 \%$ to $20 \%$.

As part of a three-year randomized trial, physicians of patients at high risk for serious illness from influenza infection in the study group received computer reminders to take preventive-care actions, while physicians of high-risk patients in the control group did not receive such reminders. Patients at risk for influenza were those over 65 years of age or with chronic lung disease, asthma, diabetes mellitus, congestive heart failure, or renal or hepatic failure.

Patients in the intervention group received influenza vaccination twice as often as patients in the control group. The potential benefits of influenza vaccination was evaluated by comparing patient outcomes in the intervention and control groups during three winters. The rates of winter morbidity, emergency room visits, and hospitalization were significantly less for patients in the intervention group than control patients.

The authors concluded that the success of computer-generated reminders should support efforts to use computerized medical records in primary practices and to include the ability to generate patientspecific preventive care reminders.

FROM: McDonald CJ, Hui SL, Tierney WM. Effects of computer reminders for influenza vaccination on morbidity during influenza epidemics. MD Computing 9;1992:304-312.

\section{NIH Opens First Trial of HIV Vaccines in Children}

The National Institutes of Health (NIH) has opened the first trial of experimental HIV vaccines in children who are infected with HIV. The trial will compare the safety of three HIV experimental vaccines in 90 HIV-infected children recruited from 12 sites nationwide.

This study will enroll children from one month to 12 years old. All volunteers must have welldocumented HIV infection but not symptoms of HIV disease other than swollen lymph glands or a mildly swollen liver or spleen. The trials will test two doses each of three experimental vaccines made from recombinant HIV proteins. These so-called subunit vaccines, each genetically engineered to contain only a piece of the virus, have so far proved well tolerated in ongoing trials in HIVinfected adults.

Preliminary evidence from similar studies under way in HIVinfected adults shows that certain vaccines can boost existing HIV-specific immune responses and stimulate new ones. It will be several years, however, before researchers know how these responses affect the clinical course of the disease. Health and Human Services Secretary Donna E. Shalala said this initial study can be seen as "a hopeful milestone in our efforts to ameliorate the tragedy of HIVinfected children who now face the certainty they will develop AIDS."

The CDC estimates that 10,000 children in the United States have HIV By the end of the decade, the World Health Organizations projects 10 million children will be infected worldwide.

In the United States, most HIV-infected children live in poor inner-city areas, and more than $80 \%$ are minorities, mainly black or Hispanic. Nearly all HIV infected children acquire the virus from their mothers during pregnancy or at birth. In the United States, an HIV-infected mother has more than a one in four chance of transmitting the virus to her baby. HIV disease progresses more rapidly in infants and children than in adults. The most recent information suggests that $50 \%$ of infants born with HIV develop a serious AIDS-related infection by three to six years of age. These infections include severe or frequent bouts of common bacterial illnesses of childhood that can result in seizures, pneumonia, diarrhea, and other symptoms leading to nutritional problems and long hospital stays.

For more information about the trial sites or eligibility for enrollment, call AIDS Clinical Trials Information Service, 1-800-TRIALS-A, from 9 A.M. to 7 P.M., EDT weekdays.

\section{Court Rules on Patient's Right to File Suit for Fear of Contracting HIV from Surgeon}

The Maryland Court of Appeals reversed the decision of a Baltimore circuit court that dismissed the claims of a patient alleging negligence and failure to obtain informed consent, fraud, and intentional infliction of emotional distress resulting from being operated on by a surgeon whom she did not know was infected with HIV

The patient learned of the surgeon's illness from a local newspaper article. The patient claimed that, as a result of the operation and the subsequent discovery of the HIV status of the surgeon, injury was incurred in the form of exposure to HIV and risk of AIDS, physical injury and financial cost from blood testing for HIV, pain, fear, anxiety, and severe emotional distress. The Baltimore circuit court, in dismissing the case in its entirety, ruled that the surgeon owed no duty to disclose his ailment as part of the doctorpatient exchange leading to informed consent and that 